diarrhea lasting $>4$ days in the first 2 trimesters, coughs, and/or vaginal yeast infection in prenatal life had an increased risk of epilepsy. Cough lasting $>1$ week was a risk factor only in the first year of life, and vaginal yeast infection only in children born preterm. Genital herpes, venereal warts, and herpes labialis were not risk factors. These associations were not changed in children with cerebral palsy $(0.2 \%)$, congenital malformation $(7.2 \%)$, or low Apgar $(<7)$ at 5 minutes (1.8\%). (Sun Y, Vestergaard M, Christensen J, Nahmias AJ, Olsen J. Prenatal exposure to maternal infections and epilepsy in childhood: a population-based cohort study. Pediatrics May 2008;121:e1100-e1107). (Respond: Yuelian Sun MD, Department of Epidemiology, University of Aarhus, Vennelyst Blvd 6, Aarhus, 8000 C, Denmark. E-mail: ys@soci.au.dk).

COMMENT. Some maternal infections are associated with an increased risk of epilepsy during childhood. The mechanisms underlying the associations are unknown, but fever and cytokines are possible factors. (Adinolfi M. Dev Med Child Neurol 1993;35:549553; Dammann O, Leviton A. Pediatr Res 1997;42:1-8).

\title{
CONGENITAL CYTOMEGALOVIRUS INFECTION AND RISK OF EPILEPSY
}

The clinical, laboratory and neuroradiological findings in 19 children with congenital cytomegalovirus (CMV) infection were retrospectively reviewed for features of epilepsy in 7 (37\%), in a study at Osaka Medical Center, Japan. Partial seizures occurred in 5 at a mean age of 20 months (range 2-37 months), West syndrome occurred in 3 patients. Seizures were uncontrolled at time of last follow-up (mean 96 months) in 6 patients. Neonatal clinical features (gestational age, gender, birth asphxia, microcephaly, chorioretinitis, neonatal seizure) were not predictive of development of epilepsy with CMV, whereas imaging abnormalities (ventricular dilatation and migration disorder) were risk factors. (Suzuki Y, Toribe Y, Mogami Y, Yanagihara K, Nishikawa M. Epilepsy in patients with congenital cytomegalovirus infection. Brain \& Dev June 2008;30:420-424). (Respond: Dr Yasuhiro Suzuki.E-mail: yasuzuki@mch.pref.osaka.jp).

COMMENT. Neuroradiographic findings, rather than clinical symptoms at birth, are most predictive of development of epilepsy in children with CMV infection. West syndrome in $43 \%$ of 7 patients in this series is a lower prevalence for this seizure type than expected.

\section{HERPES SIMPLEX VIRUS TYPE 2 NEUROLOGIC COMPLICATIONS}

The neurologic complications of HSV-2 infection are reviewed by researchers at University of Kentucky College of Medicine, Lexington. HSV-2-associated neurologic disease results from primary infection or reactivation of latent HSV-2. Primary infection occurs in neonates but is usually delayed until adolescence and adulthood, following sexual activity. HSV-2 latency and reactivation is centered in sacral ganglia, but may also be widespread in the CNS. Approximately $90 \%$ of infections are unrecognized. Neurological complications of HSV-2 infection involve any part of the neuraxis. Encephalitis (HSE) is the most frequent manifestation of HSV-2 in neonates, and onset is heralded by focal or generalized seizures. CSF shows a lymphocytic pleocytosis, increased protein, and PCR 